\title{
Optimizing the Treatment Mode for De Novo Metastatic Nasopharyngeal Carcinoma With Bone- only Metastasis
}

Cheng Lin ( $\square$ happygo1988@sina.com )

Fujian Medical University Cancer Hospital \& Fujian Cancer Hospital

Sheng Lin

Fuqing city hospital of Fujian

lili Zhu

Fuzhou Pulmonary hospital of Fujian

JianJi Pan

Fujian Medical University Cancer Hospital, Fujian Cancer Hospital

Yun Xu

Fujian Medical University Cancer Hospital \& Fujian Cancer Hospital shaojun lin

Fujian Medical University Cancer Hospital \& Fujian Cancer Hospital

\section{Research Article}

Keywords: metastatic nasopharyngeal carcinoma, palliative chemotherapy, locoregional radiotherapy, local radiotherapy, bone metastasis, overall survival

Posted Date: September 22nd, 2021

DOI: https://doi.org/10.21203/rs.3.rs-685930/v2

License: (c) (i) This work is licensed under a Creative Commons Attribution 4.0 International License. Read Full License

Version of Record: A version of this preprint was published at BMC Cancer on January 4th, 2022. See the published version at https://doi.org/10.1186/s12885-021-09152-1. 


\section{Abstract}

Purpose: No standard radiotherapy regimens was established in the treatment of de novo metastatic nasopharyngeal carcinoma (mNPC) with bone-only metastasis. The current study aimed to investigate the efficacy of palliative chemotherapy (PCT) plus locoregional radiotherapy (LLRT) with or without local radiotherapy (RT) to bone metastatic lesions in $\mathrm{MNPC}$, and identify the optimal candidates.

Methods: We retrospectively analyzed 141 de novo mNPC patients with bone-only metastasis who received at least two cycles of PCT with or without LLRT and RT to bone metastasis. The difference in survival was evaluated by the log-rank test. Univariable and multivariable analysis was made by Cox regression.

Results: Patients who received PCT plus LLRT had significantly longer overall survival (OS) (45.0 months vs 13.5 months, $\mathrm{HR}=0.30, p=0.001)$ and progression-free survival (PFS) (29.0 months vs 11.0 months, $\mathrm{HR}=0.34, p=0.014$ ), especially in patients who had less than 3 metastatic bone lesions. Multivariate analysis confirmed that LRRT, more chemotherapy cycles $(\geq 4)$ and limited number of bone metastasis $(\leq 3)$ were favorable prognostic factors for OS. Subgroup analysis revealed that RT to metastatic bones had a tendency to prolong the survival time in the unselected population who received PCT plus LLRT ( $p>$ 0.05 ), while further data suggested that RT to metastatic bones dramatically improve OS (72.0 months vs 26.0 months, $p=0.002)$ and PFS $(60.0$ months vs 20.0 months, $p=0.006)$ for mNPC with less than 3 metastatic bone lesions.

Conclusions: LLRT and RT to bone metastatic lesions followed by PCT in de novo mNPC with bone-only metastasis significantly prolonged survival in patients with less than 3 metastatic bone lesions.

\section{Introduction}

Nasopharyngeal carcinoma is an endemic malignancy in southern China, with an incidence of up to 30 cases per 100000 person-years[1], and approximately $4-10 \%$ of those patients were metastatic NPC ( $\mathrm{mNPC}$ ) at diagnosis [2]. mNPC is a heterogeneous entity that ranges from a single metastasis to multiple organ metastases. Bone metastasis is the mostly common invaded organ, accounting for over $60 \%$ of all metastatic sites and favoring longer survival[3]. To date, mNPC were generally considered incurable and there is no optional treatment. Palliative chemotherapy (PCT) is the primary treatment, locoregional radiation therapy (LLRT) is strongly recommended in chemotherapy-sensitive patients with mNPC $[4,5]$. While radiotherapy (RT) to metastatic bones is only widely administrated for relieving pain and improving quality of life in de novo mNPC with bone metastasis.

Nowadays, increasing studies had reported that NPC patients with solitary bone metastasis, or even with recurrent bone-only oligometastatic could have a long-term disease control and a better survival $[6,7]$. Moreover, emerging evidences have suggested a vital role of local radiotherapy in de novo MNPC with bone-only metastasis, giving a fascinating insight into the management of bone metastatic mNPC [811]. However, the potential benefit of combine PCT plus LLRT with or without RT to metastatic bones in 
mNPC remains controversial. No general consensus was established and no standard regimens was strongly recommended $[2,4]$.

In the present study, we retrospectively analyzed 141 de novo mNPC with bone-only metastasis between June 2007 and December 2017 at our cancer center, and explored the clinical significance of different practice strategy in de novo mNPC with different patterns of bone metastasis (metastatic bone sites $\leq 3$ and $>3$ ), and aimed to optimize the treatment regimens and found the most potential candidates.

\section{Materials And Methods}

\section{Patients}

141 patients with mNPC who were admitted to Fujian Cancer Hospital between June 2007 and December 2017. Inclusion criteria were as follows: (I) patients were newly and histologically diagnosed with mNPC; (II) mNPC with bone-only metastasis. Exclusion criteria were as follows: (I) patients with NPC developed multiple organ metastases; (II) patients who were previously treated; (III) patients who were lost in the follow up; (IV) patients had less than two cycle of chemotherapy. Re-staging of all patients was done according to the 8th edition of American Joint Committee on Cancer (AJCC) / Union for International Cancer Control (UICC). Our study was approved by the Ethics Committee of Fujian Medical University Cancer Hospital, Fuzhou, China. Written informed consent was obtained from all patients.

\section{Treatment}

All patients received platinum-based systematic chemotherapy. Chemotherapy regimens, including gemcitabine, paclitaxel or docetaxel plus platinum, were administered every 3-4 weeks. LRRT to nasopharynx and neck was conducted by two-dimensional radiotherapy (2DRT) or intensity modulated radiotherapy (IMRT), which was described as previous[12]. The patterns of radiotherapy to metastatic bone were heterogeneous. 40 Gy with 20 fractions or 30Gy with 10 fractions were most commonly used, and few patients received 50 Gy using 25 fractions or 60-70 Gy.

\section{Follow-up}

Evaluation of tumor response, including computed tomography (CT), magnetic resonance imaging, emission computed tomography or positron emission tomography CT, was selectively conducted after every two or three cycles of chemo therapy. After all therapeutic process, patients were evaluated every 3 months for the first 2 years, every 6 months from year $3-5$, and then every 12 months. The overall survival (OS) was measured from the date of diagnosis to the date of death from any cause. The progression-free survival (PFS) was measured from the date of diagnosis to the time of treatment failure.

\section{Statistical analysis}


All statistical analyses were performed using the soft-ware SPSS version 24.0 and Graph Pad Prism 8. The Cox regression model was used for the univariate analysis and multivariate analysis. The propensity score matching (PSM) analysis was used to reduce the data bias and the confounding variable. KaplanMeier analysis and log-rank method were used to compare survival difference. $p$ values $<0.05$ were considered statistically significant, and all $p$ values were two sided.

\section{Results}

\section{Patient characteristics}

A total of 141 de novo mNPC patients with bone-only metastasis who were treated with PCT with or without LLRT and RT to metastatic bones between January 2007 and December 2017 were eligible for our study (Fig. 1).

The median age was 46.1 years (range 17-73 years). With a median follow-up time of 71.5 months (95\% $\mathrm{Cl}, 57.6-85.4$ months). The median OS was 38.0 months ( $95 \% \mathrm{Cl}, 24.4-51.6$ months), and the 1-, 3- and 5- year survival rate was $100 \%, 88.7 \%$ and $82.2 \%$ respectively (Fig. 2 ).

The comparison of patients' characteristics between PCT plus LLRT and PCT alone were shown in (Table 1). 
Table 1

Characteristics of 141 de novo mNPC with Bone-only metastasis

\begin{tabular}{|c|c|c|c|}
\hline \multirow[t]{2}{*}{ Characteristics } & PCT plus LLRT & PCT alone & $p$ \\
\hline & No $(\%)(n=131)$ & No $(\%)(n=10)$ & \\
\hline Age(y) & & & 0.502 \\
\hline$\leq 50$ & $84(64.1)$ & $8(80.0)$ & \\
\hline$>50$ & $47(35.9)$ & $2(20.0)$ & \\
\hline Sex & & & 0.815 \\
\hline Female & $107(81.7)$ & $9(90.0)$ & \\
\hline Male & $24(18.3)$ & $1(10.0)$ & \\
\hline T stage & & & 0.104 \\
\hline T1-2 & $39(29.8)$ & $6(60.0)$ & \\
\hline T3-4 & $92(70.2)$ & $4(40.0)$ & \\
\hline $\mathrm{N}$ stage & & & 0.907 \\
\hline N0-1 & $22(16.7)$ & $1(10.0)$ & \\
\hline N2-3 & $110(83.3)$ & $9(90.0)$ & \\
\hline ECOG score & & & 1.000 \\
\hline 0 & $118(90.1)$ & $9(90.0)$ & \\
\hline 1 & $13(9.9)$ & $1(10.0)$ & \\
\hline Chemo cycles & & & 0.417 \\
\hline$<4$ & $42(30.5)$ & $5(50.0)$ & \\
\hline$\geq 4$ & $77(60.0)$ & $5(50.0)$ & \\
\hline No. of bone metastasis & & & 0.480 \\
\hline$\leq 3$ & $44(33.6)$ & $5(50.0)$ & \\
\hline$>3$ & $87(66.4)$ & $5(50.0)$ & \\
\hline RT to bone metastasis & & & 0.419 \\
\hline No & $81(61.8)$ & $8(80.0)$ & \\
\hline Yes & $50(38.2)$ & $2(20.0)$ & \\
\hline
\end{tabular}


Majority of NPC patients received PCT plus LLRT (92.9\%), among them, 44 patients had less than 3 bone metastatic lesions. $37.9 \%$ of all populations received RT to bone metastasis in PCT plus LLRT group.

\section{Efficacy Of Additional Llit}

Kaplan-Meier curves showed that median OS in the PCT plus LLRT group was significantly longer than the PCT alone group ( 45.0 months vs 13.5 months; $p=0.001$ ) (Fig. $3 a)$, as well as PFS ( 29.0 months vs 11.0 months; $p=0.014$ ) (Fig. $3 b$ ). In order to confirm whether LRRT can benefit all patients who received PCT plus LLRT, we conducted a subgroup analysis based on the number of bone metastasis. Patients who had less than 3 bone metastatic lesions were closely associated with longer median OS than those who had more than 3 bone metastatic lesions (63.5 months vs 24.0 months; $p<0.001$ ) (Fig. 3c), which was also tanable in term of the median PFS (48.0 months vs 16.0 months; $p=0.010)$ (Fig. 3d).

Univariate and Multivariate analysis further confirmed LRRT was an independent prognostic factor in OS for de novo mNPC patients (Table 2). In addition, Multivariate analysis also suggested that more chemotherapy cycles $(\geq 4)$ and limited bone metastasis predict better survival outcome. 
Table 2

Univariable and Multivariate analysis for PFS and OS in 141 de novo MNPC patients

\begin{tabular}{|c|c|c|c|c|}
\hline & \multicolumn{2}{|l|}{ Univariable } & \multicolumn{2}{|l|}{ Multivariable } \\
\hline & $\mathrm{HR}(95 \% \mathrm{Cl})$ & $p$ & $\mathrm{HR}(95 \% \mathrm{Cl})$ & $p$ \\
\hline \multicolumn{5}{|l|}{ Progress-free survival } \\
\hline Age ( $\leq 50$ vs > 50) & $\begin{array}{l}1.030(0.655- \\
1.620)\end{array}$ & 0.898 & & \\
\hline Sex (Female vs Male) & $\begin{array}{l}0.931(0.532- \\
1.629)\end{array}$ & 0.801 & & \\
\hline Chemotherapy cycles (<4 vs $\geq 4$ ) & $\begin{array}{l}0.325(0.208- \\
0.507)\end{array}$ & $\begin{array}{l}< \\
0.001\end{array}$ & $\begin{array}{l}0.363(0.230- \\
0.573)\end{array}$ & $<0.001$ \\
\hline $\begin{array}{l}\text { Locoregional radiation therapy (No } \\
\text { vs Yes) }\end{array}$ & $\begin{array}{l}0.479(0.230- \\
0.999)\end{array}$ & 0.050 & $\begin{array}{l}0.712(0.338- \\
1.501)\end{array}$ & 0.187 \\
\hline No. of bone metastasis ( $\leq 3$ vs $>3$ ) & $\begin{array}{l}0.517(0.333- \\
0.802)\end{array}$ & 0.003 & $\begin{array}{l}0.597(0.383- \\
0.930)\end{array}$ & 0.023 \\
\hline \multicolumn{5}{|l|}{ Overall Survival } \\
\hline Age $(\leq 50$ vs $>50)$ & $\begin{array}{l}1.404(0.904- \\
2.181)\end{array}$ & 0.131 & & \\
\hline Sex (Female vs Male) & $\begin{array}{l}0.993(0.575- \\
1.715)\end{array}$ & 0.981 & & \\
\hline Chemotherapy cycles (< 4 vs $\geq 4$ ) & $\begin{array}{l}0.298(0.193- \\
0.460)\end{array}$ & $\begin{array}{l}< \\
0.001\end{array}$ & $\begin{array}{l}0.337(0.217- \\
0.523)\end{array}$ & $<0.001$ \\
\hline $\begin{array}{l}\text { Locoregional radiation therapy (No } \\
\text { vs Yes) }\end{array}$ & $\begin{array}{l}0.335(0.166- \\
0.676)\end{array}$ & 0.002 & $\begin{array}{l}0.439(0.216- \\
0.891)\end{array}$ & 0.023 \\
\hline No. of bone metastasis ( $\leq 3$ vs $>3$ ) & $\begin{array}{l}0.473(0.305- \\
0.732)\end{array}$ & 0.001 & $\begin{array}{l}0.536(0.345- \\
0.832)\end{array}$ & 0.005 \\
\hline
\end{tabular}

\section{Efficacy of additional RT to metastatic bones in patients received PCT plus LLRT}

To explore whether RT to metastatic bones would generate actual benefits in de novo mNPC patients who had already received PCT plus LLRT, Kaplan-Meier analysis was conducted. The data revealed that despite the trend of benefit, RT to metastatic bones had no statistical significance in OS (60.0 months vs 38.0 months, $p=0.492$ ) (Fig. 4a) and PFS (37.0 months vs 25.0 months, $p=0.503$ ) (Fig. 4b ).

To eliminate the unbalanced factors between the above two groups, propensity score matching (PSM) (1:1) with a nearest neighbor was performed (Additional file 1: Table S1).

The Kaplan-Meier demonstrated that RT to metastatic bones was not closely associated with better OS and PFS ( $p>0.05)$ (Additional file 2: Figure S1). 
In order to screen the dominant population of RT to metastatic bones, subgroup analysis in 50 patients who received PCT plus LLRT and RT to metastatic bones was done. Patients was stratified by number of metastatic bone sites ( $\leq 3$ group and $>3$ group), as we had found number of bone metastasis was an independent prognostic factor in OS and PFS. Interestingly, Kaplan-Meier analysis showed that RT to metastatic bones had significantly longer median OS (72.0 months vs 26.0 months, $p=0.002)$ (Fig. 4c) and PFS (60.0 months vs 20.0 months, $p=0.006)$ (Fig. 4d).

\section{Discussion}

Treatment of mNPC was a major challenge for radiotherapy physician. The skeleton was the most common site of distant metastasis in NPC, whereas the optimal therapeutic strategy has remained largely undefined. Our study showed that compared to PCT alone, combination of PCT and LLRT could improve OS and PFS in de novo mNPC with bone-only metastasis, especially in patients with limited metastatic bone sites $(\leq 3)$. Moreover, LLRT and number of bone metastasis $(\leq 3)$ were favorable independently factor for OS. For patients receiving PCT plus LLRT, there was no significant benefit from RT to metastatic bones in unselected patients. Subgroup analysis further confirmed that RT for metastatic bones only improve OS and PFS for mNPC with less than 3 metastatic bone lesions.

Local radiotherapy in $\mathrm{mNPC}$ is becoming hot-button issues. Rusthoven et al. reported that compared to chemotherapy alone, local radiotherapy combined with chemotherapy was associated with improved OS (median OS 21.4 vs 15.5 months, 5 -year OS $28 \%$ vs 10\%), resulting in a $39 \%$ reduction of death risk[13]. Similar findings were also found in some retrospective studies[14-20]. Up to now, there are only one multicenter phase 3 randomized clinical trial investigated the efficacy of LLRT in de novo mNPC. The trial demonstrated that PCT plus LLRT can significantly prolong OS of chemotherapy-sensitive mNPC patients. The 2-year survival rate was $76.4 \%$ in PCT plus LRRT group versus $54.5 \%$ in the PCT alone group ( $\mathrm{HR}=0.42)$ [5]. As to mNPC with bone-only metastasis, Shen et al found that compared with chemotherapy or radiotherapy alone, combined chemoradiotherapy could significantly benefit patients with single bone metastasis $(H R=0.21)$. In additional, spinal metastasis and more than 3 bone metastasis sites are unfavorable elements for OS [21]. Consistent with above studies, our study also highlight the significance of LLRT in de novo MNPC with bone-only metastasis. Our study suggest that patients receiving PCT plus LLRT had over three times OS than PCT alone (45.0 months vs 13.5 months), and longer OS was remarkably observed in patients who had less than 3 bone ( median OS $=63.5$ months). While our results were somewhat different from Shen' study, our study suggested that less chemotherapy cycles ( $<4)$, no locoregional radiation therapy and larger number of bone metastasis $(>3)$ were associated with worse OS. Taken together, LLRT should be considered after PCT in clinical practice of mNPC in future, especially for mNPC patients with bone-only metastasis. More randomized clinical trials are warranted.

For mNPC patients with bone-only metastasis, who will benefit from RT to metastatic bones has not yet been well characterized. Li et al reported that patients who received intensive local radiotherapy to bone lesions had longer OS $(H R=0.63)$ and PFS $(H R=0.80)$, and the post-treatment EBV DNA level and radical 
radiation dose were independent prognostic factors for OS [9]. However, our study indicated that for patients receiving PCT plus LLRT, the benefit from RT to metastatic bones was only seen in MNPC with less than 3 metastatic bone lesions, not in patients without selection. Of note, patients receiving PCT plus LLRT and RT to metastatic bones lives the longest survival. Therefore, for mNPC with less than 3 metastatic bone lesions, radical radiotherapy to metastatic bone would be strongly preferred as patients may have a long-term survival.

Since there is no consensus on who should receive LLRT and / or RT to metastatic sizes after first-line PCT, prognostic models and risk stratifications guiding the subdivisions of mNPC patients based on clinical features, therapeutic response and blood biomarkers had been widely researched to identify the optimal candidates $[3,14,17,19,22-25]$, as well as in mNPC with bone-only metastasis[6, 9-11, 21, 26].

Li et al suggested that instead of high-risk mNPC, low-risk patients experienced significant survival benefits from definitive radiation therapy in addition to palliative chemotherapy (PCT) based on 5 prognostic factors, including response of metastases to chemotherapy, number of metastatic sites, liver metastasis, serum lactate dehydrogenase and posttreatment EBV-DNA [22]. Zou et al reported PCT combined with LLRT may benefit mNPC patients without liver involvement liver involvement [3]. Xu et al found that additional LRRT after PCT may only improve OS for oligometastatic patients, rather than polymetastatic disease [23]. Similar findings were also seen in other studies [15, 24]. With respect to the mNPC with bone-only metastasis, chen et al. develop a prognostic score and divided patients into low and high risk groups based on age, N, anemia, bone metastasis free interval, without radiotherapy to primary sites and without radiotherapy to first metastasis sites [26]. Sun et al. subdivide MNPC with boneonly metastasis based on EBV DNA after PCT and the number of metastatic lesions[10]. Our study classified mNPC into two group through number of bone metastatic lesions, proving a simple and feasible way to predict survival and guide clinical practice. In summary, looking for reliable and valuable biomarkers is vital to aid doctors in selecting the most suitable patients for individualized and comprehensive therapy.

The study had sone limitations. Firstly, our study is retrospective study in single-center. Secondly, our simple sizes are relatively small, which might affect statistical performance even when PSM was performed. Thirdly, EBV DNA and other blood biomarkers were not assessed in our study. Further prospective trails are needed in the future to guide the management of de novo MNPC with bone-only metastasis.

\section{Conclusions}

These findings suggest that PCT plus LLRT and RT to metastatic bones really remarkably improves survival in de novo mNPC with limited number of bone metastasis $(n \leq 3)$. Our study supports an aggressive interventions for selective mNPC patients with bone-only metastasis. Prospective clinical trials are expected to confirm these results and to find the optimal population.

\section{Abbreviations}


RT: radiation therapy; LRRT: locoregional radiation therapy; mNPC:metastatic nasopharyngeal carcinoma; PCT: palliative chemotherapy; IMRT: intensity-modulated radiotherapy; OS: Overall survival; PFS: progression-free survival; PSM: Propensity score-matched; ECOG: Eastern Cooperative Oncology Group; AJCC: American Joint Committee on Cancer; UICC: Union for International Cancer Control; HR: Hazard ratios; $\mathrm{Cl}$ : confidence interval; EBV: Epstein-Barr virus.

\section{Declarations}

\section{Ethical approval and consent to participate}

This study was approved by the Ethics Committee of Fujian Medical University Cancer Hospital, Fuzhou, China.

\section{Consent for publication}

Not applicable.

\section{Availability of data and material}

The datasets generated and/or analyzed in our study are available from the corresponding author.

\section{Competing interests}

The authors declare that they have no competing interests.

\section{Ethical statement and consent to participate}

The study protocol was designed in accordance with the guidelines outlined in the Declaration of Helsinki. All information was retrospectively extracted in the context of compliance with the relevant regulations and protection of patients' privacy. This study was approved by the Ethical Review Committee of Fujian Cancer Hospital (No. SQ2019-031-01). All of the participants signed an informed consent form.

\section{Funding}

This study was supported by Joint Funds for the Innovation of Science and Technology \&Fujian Province هGrant number: 2019Y9037区, Natural Science Foundation of Fujian Province (Grant number:

2020J05071), Fujian Province Health Middle-aged and Young backbone personnel training Project (Grant number: 2020GGB010) and the Startup Fund for scientific research, Fujian Medical University $₫$ Grant number: 2019QH1192Х.

\section{Authors' contributions}

All authors helped to perform the research; LC participated in manuscript writing and data analysis; PJJ, LSJ and LC participated in study concept and study design. LS and ZLL participated in data collection. All authors approved the final manuscript. 
None.

\section{References}

1. Wei WI, Sham JS: Nasopharyngeal carcinoma. Lancet 2005, 365(9476):2041-2054.

2. Chen Y-P, Chan ATC, Le Q-T, Blanchard P, Sun Y, Ma J: Nasopharyngeal carcinoma. The Lancet 2019, 394(10192):64-80.

3. Zou X, You R, Liu H, He YX, Xie GF, Xie ZH, Li JB, Jiang R, Liu LZ, Li L et al: Establishment and validation of $\mathrm{M} 1$ stage subdivisions for de novo metastatic nasopharyngeal carcinoma to better predict prognosis and guide treatment. Eur $\mathrm{J}$ Cancer 2017, 77:117-126.

4. Bossi P, Chan AT, Licitra L, Trama A, Orlandi E, Hui EP, Halamkova J, Mattheis S, Baujat B, Hardillo J et al: Nasopharyngeal carcinoma: ESMO-EURACAN Clinical Practice Guidelines for diagnosis, treatment and follow-up(dagger). Ann Oncol 2021, 32(4):452-465.

5. You R, Liu YP, Huang PY, Zou X, Sun R, He YX, Wu YS, Shen GP, Zhang HD, Duan CY et al: Efficacy and Safety of Locoregional Radiotherapy With Chemotherapy vs Chemotherapy Alone in De Novo Metastatic Nasopharyngeal Carcinoma: A Multicenter Phase 3 Randomized Clinical Trial. JAMA Oncol 2020, 6(9):1345-1352.

6. Khot A, Love C, Garg MK, Haigentz M, Jr.: Long-Term Disease Control in a Patient With Recurrent Bone-Only Oligometastatic Nasopharyngeal Carcinoma. J Clin Oncol 2016, 34(4):e25-26.

7. Lim A, Corry J, Lau E, Rischin D: Prolonged remission in a patient with nasopharyngeal carcinoma with a solitary bone metastasis. J Clin Oncol 2011, 29(6):e135-137.

8. Cao X, Han Y, He L, Xiang J, Wen Z: Risk subset of the survival for nasopharyngeal carcinoma patients with bone metastases: who will benefit from combined treatment? Oral Oncol 2011, 47(8):747-752.

9. Li XY, Jia GD, Sun XS, Guo SS, Liu LT, Liu SL, Yan JJ, Luo DH, Sun R, Guo L et al: Intensive Local Radiotherapy Is Associated With Better Local Control and Prolonged Survival in Bone-Metastatic Nasopharyngeal Carcinoma Patients. Front Oncol 2020, 10:378.

10. Sun XS, Liang YJ, Liu SL, Chen QY, Guo SS, Wen YF, Liu LT, Xie HJ, Tang QN, Li XY et al: Subdivision of Nasopharyngeal Carcinoma Patients with Bone-Only Metastasis at Diagnosis for Prediction of Survival and Treatment Guidance. Cancer Res Treat 2019, 51(4):1259-1268.

11. Nong S, Pan X, Chen K, Li Y, Zhu X: Therapeutic Effect of Chemotherapy Cycle in Nasopharyngeal Carcinoma (NPC) Patients Who Developed Bone-Only Metastasis. Med Sci Monit 2020, 26:e922244.

12. Lin C, Lin S, Guo Q-J, Zong J-F, Lu T-Z, Lin N, Lin S-J, Pan J-J: Systemic immune-inflammation index as a prognostic marker in patients with newly diagnosed metastatic nasopharyngeal carcinoma: a propensity score-matched study. Translational Cancer Research 2019, 8(5):2089-2098. 
13. Rusthoven CG, Lanning RM, Jones BL, Amini A, Koshy M, Sher DJ, Bowles DW, McDermott JD, Jimeno A, Karam SD: Metastatic nasopharyngeal carcinoma: Patterns of care and survival for patients receiving chemotherapy with and without local radiotherapy. Radiother Oncol 2017, 124(1):139-146.

14. Sun XS, Liu LT, Liu SL, Guo SS, Wen YF, Xie HJ, Tang QN, Liang YJ, Li XY, Yan JJ et al: Identifying optimal candidates for local treatment of the primary tumor among patients with de novo metastatic nasopharyngeal carcinoma: a retrospective cohort study based on Epstein-Barr virus DNA level and tumor response to palliative chemotherapy. BMC Cancer 2019, 19(1):92.

15. Hu SX, He XH, Dong M, Jia B, Zhou SY, Yang JL, Yang S, Zhang CG, Liu P, Qin Y et al: Systemic chemotherapy followed by locoregional definitive intensity-modulated radiation therapy yields prolonged survival in nasopharyngeal carcinoma patients with distant metastasis at initial diagnosis. Med Oncol 2015, 32(9):224.

16. Chen MY, Jiang R, Guo L, Zou X, Liu Q, Sun R, Qiu F, Xia ZJ, Huang HQ, Zhang L et al: Locoregional radiotherapy in patients with distant metastases of nasopharyngeal carcinoma at diagnosis. Chin $\mathrm{J}$ Cancer 2013, 32(11):604-613.

17. Liao W, He J, Gou Q, Duan B, Liu L, Ai P, Li Y, Ren K, Chen N: Synchronous Metastatic Nasopharyngeal Carcinoma: Characteristics and Survival of Patients Adding Definitive Nasopharyngeal-Neck Radiotherapy to Systematic Chemotherapy. Cancer Manag Res 2020, 12:10211-10219.

18. Lin S, Tham IW, Pan J, Han L, Chen Q, Lu JJ: Combined high-dose radiation therapy and systemic chemotherapy improves survival in patients with newly diagnosed metastatic nasopharyngeal cancer. Am J Clin Oncol 2012, 35(5):474-479.

19. Zeng L, Tian YM, Huang Y, Sun XM, Wang FH, Deng XW, Han F, Lu TX: Retrospective analysis of 234 nasopharyngeal carcinoma patients with distant metastasis at initial diagnosis: therapeutic approaches and prognostic factors. PLoS One 2014, 9(9):e108070.

20. Yin Z, Zhang X, Wang Y, Wang P, Yuan Z: The combination of systemic therapy and locoregional radiotherapy prolongs survival in newly diagnosed metastatic nasopharyngeal carcinoma patients. Onco Targets Ther 2017, 10:5677-5683.

21. Shen L, Dong J, Li S, Wang Y, Dong A, Shu W, Wu M, Pan C, Xia Y, Wu P: M1 stage subdivision and treatment outcome of patients with bone-only metastasis of nasopharyngeal carcinoma. Oncologist 2015, 20(3):291-298.

22. Li WZ, Lv SH, Liu GY, Liang H, Guo X, Lv X, Liu KY, Qiang MY, Chen X, Gu SZ et al: Development of a Prognostic Model to Identify the Suitable Definitive Radiation Therapy Candidates in de Novo Metastatic Nasopharyngeal Carcinoma: A Real-World Study. Int J Radiat Oncol Biol Phys 2021, 109(1):120-130.

23. Xu H, Lu L, Lu T, Xu Y, Zong J, Huang C, Lin F, Zheng Y, Lin C, Lin S et al: Identifying the optimal candidates for locoregional radiation therapy in patients with de novo metastatic nasopharyngeal carcinoma. Head Neck 2021. 
24. Sun XS, Liang YJ, Chen QY, Guo SS, Liu LT, Sun R, Luo DH, Tang LQ, Mai HQ: Optimizing the Treatment Pattern for De Novo Metastatic Nasopharyngeal Carcinoma Patients: A Large-Scale Retrospective Cohort Study. Front Oncol 2020, 10:543646.

25. Yang JH, Sun XS, Xiao BB, Liu LT, Guo SS, Liang JD, Jia GD, Tang LQ, Chen QY, Mai HQ: Subdivision of de-novo metastatic nasopharyngeal carcinoma based on tumor burden and pretreatment EBV DNA for therapeutic guidance of locoregional radiotherapy. BMC Cancer 2021, 21(1):534.

26. Chen C, Wu JB, Jiang H, Gao J, Chen JX, Pan CC, Shen LJ, Chen Y, Chang H, Tao YL et al: A Prognostic Score for Nasopharyngeal Carcinoma with Bone Metastasis: Development and Validation from Multicenter. J Cancer 2018, 9(5):797-806.

\section{Figures}

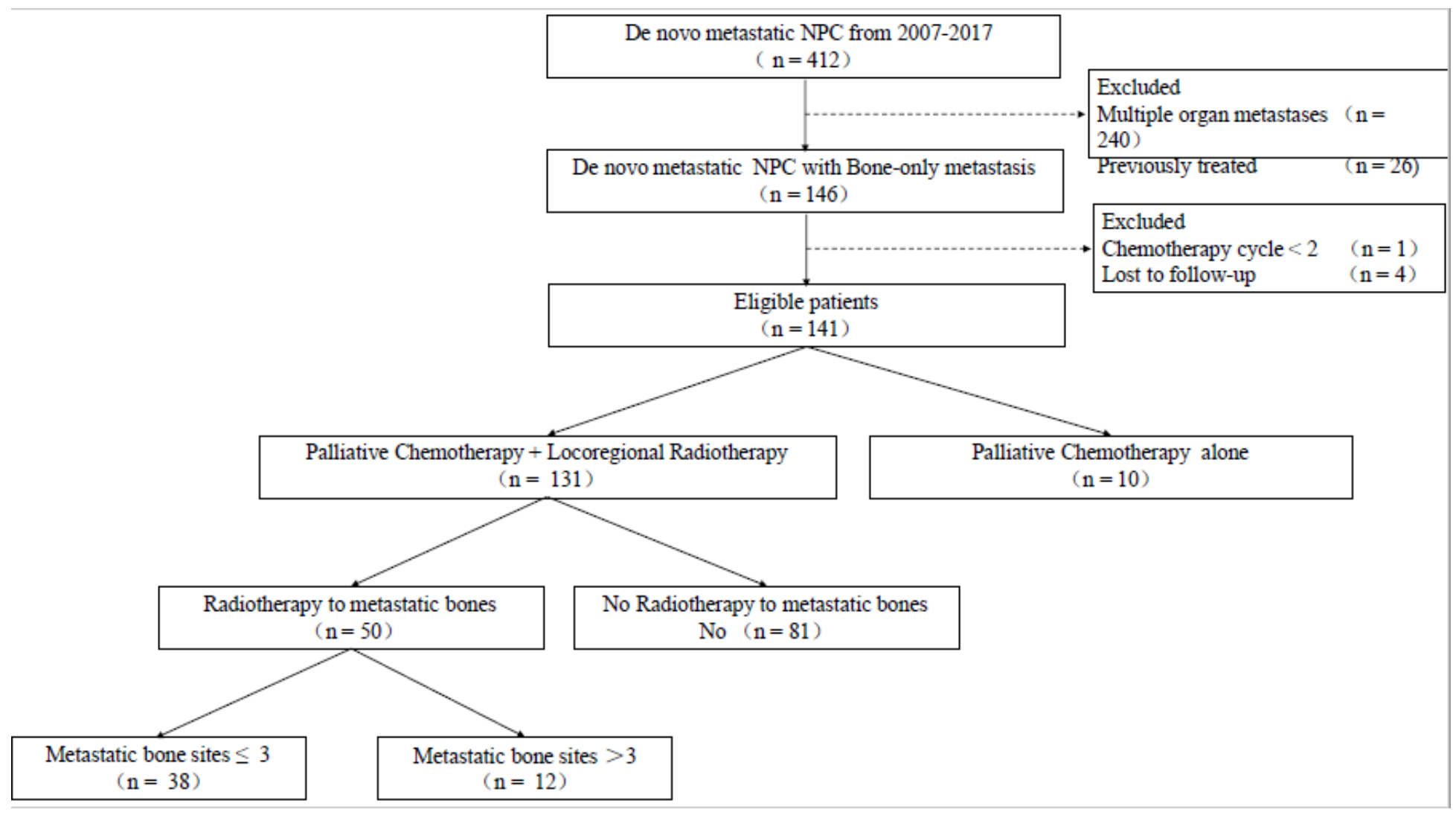

\section{Figure 1}

Flow diagram of study selection process. 


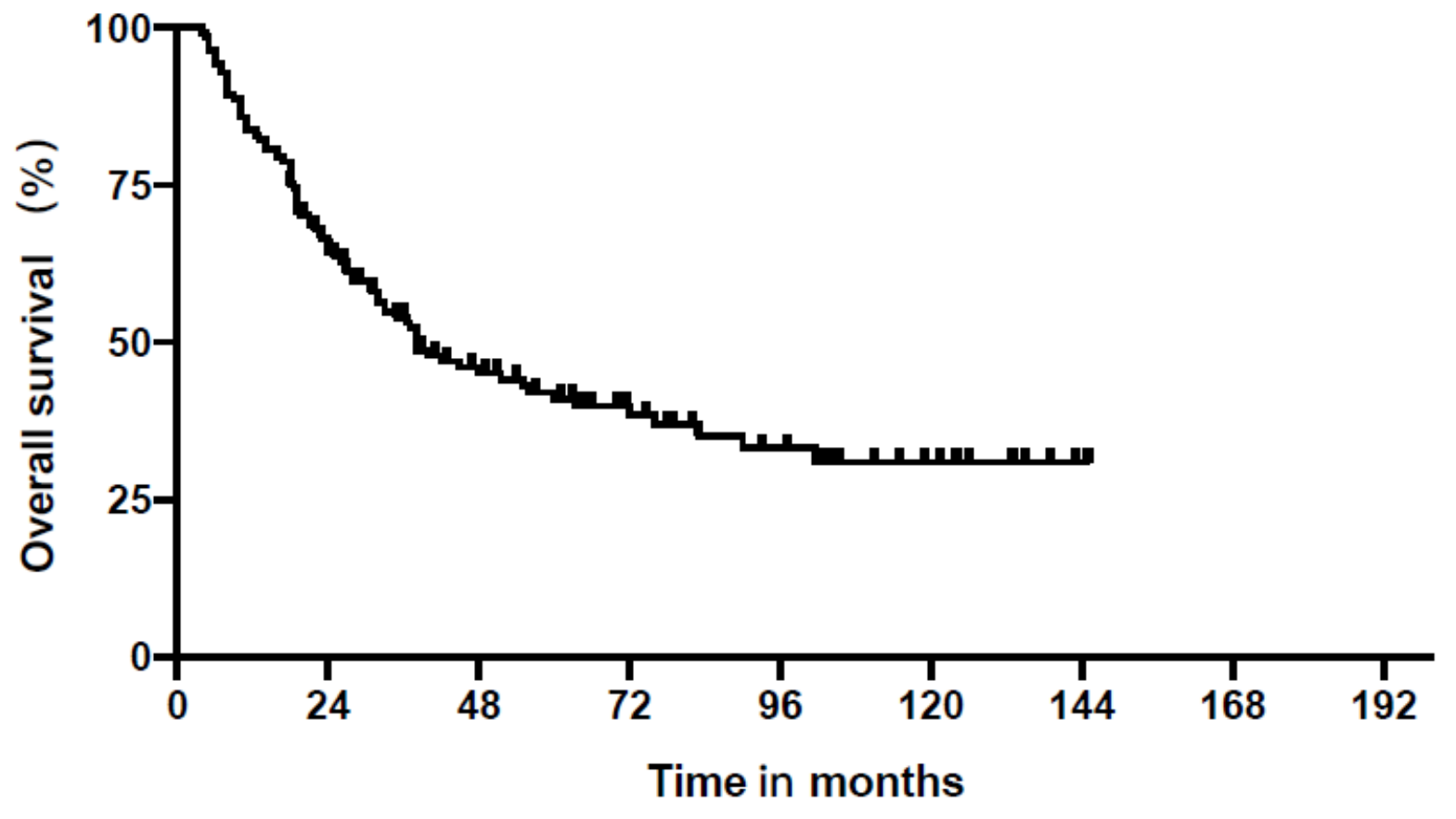

$\begin{array}{llllllll}\text { Number at risk } & 141 & 90 & 49 & 28 & 16 & 8 & 1\end{array}$

Figure 2

Kaplan-Meier curves of OS in 141 de novo mNPC patients with bone-only metastasis 
A

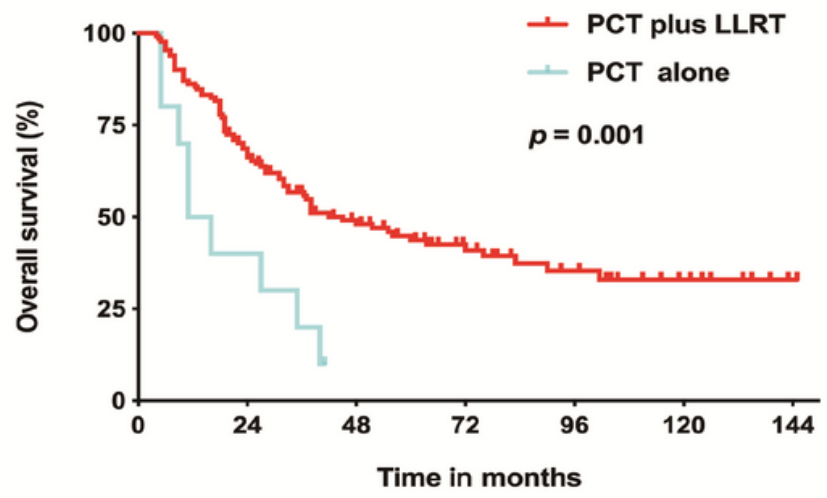

Number at risk

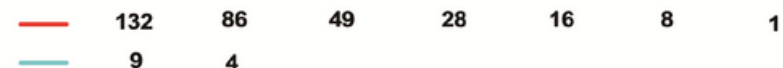

C

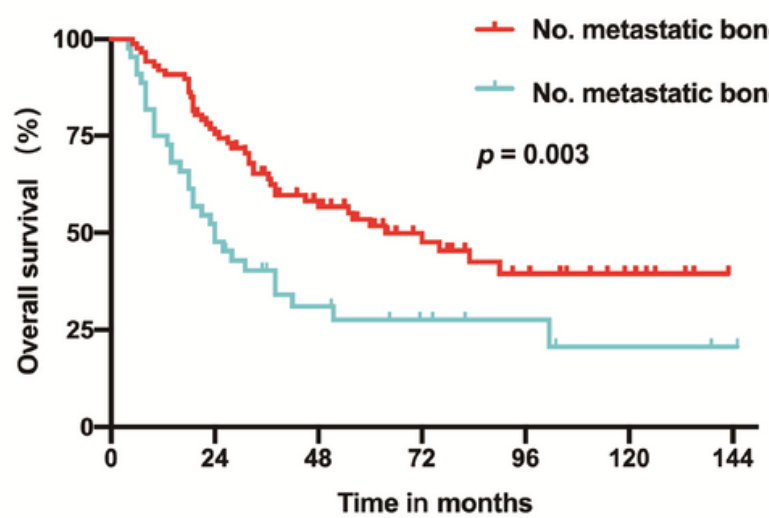

Number at risk

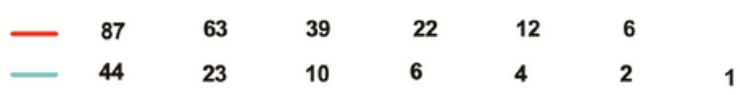

B

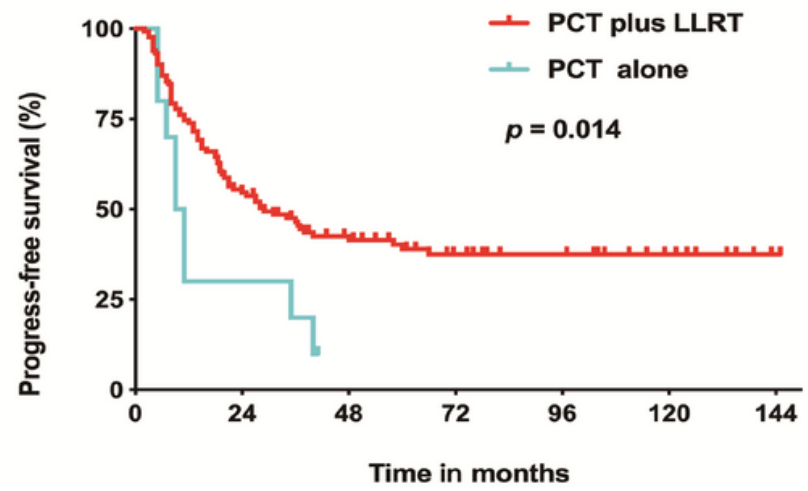

Number at risk

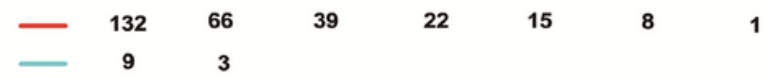

D

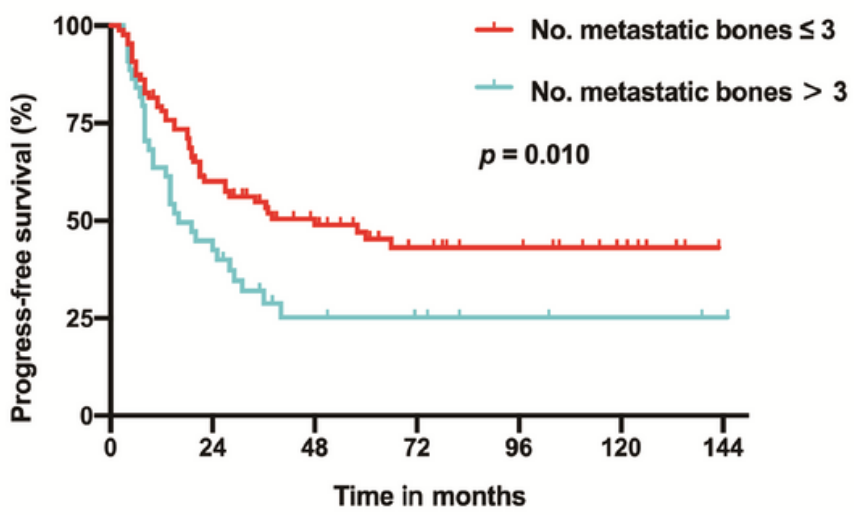

Number at risk

$\begin{array}{rrrrrrr}87 & 47 & 32 & 17 & 12 & 6 & \\ -\quad 44 & 19 & 7 & 5 & 3 & 2 & 1\end{array}$

Figure 3

Kaplan-Meier curves for OS (a) and PFS (b) of 141 de novo mNPC patients receiving at least two cycles palliative chemotherapy (PCT); Kaplan-Meier curves according to No. of metastatic bones for OS (c) and PFS (d) of 131 de novo mNPC patients with bone-only metastasis receiving PCT and locoregional radiation therapy (LLRT). 
A

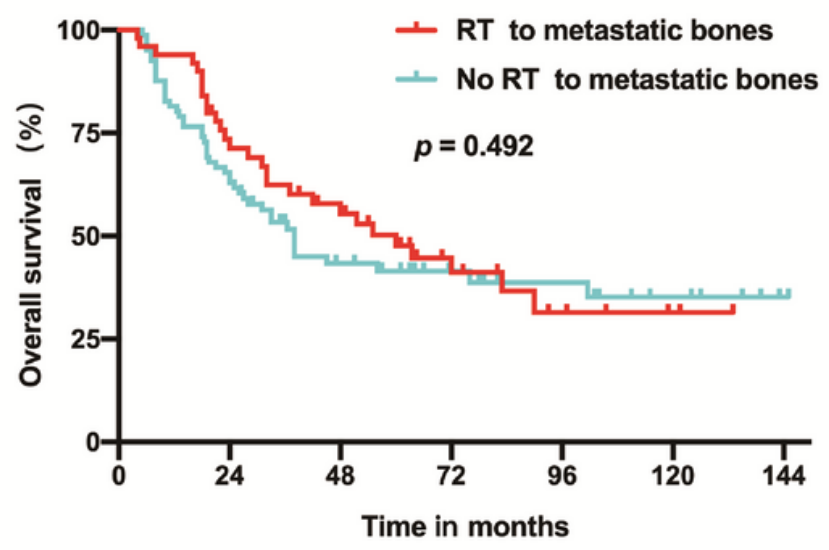

C

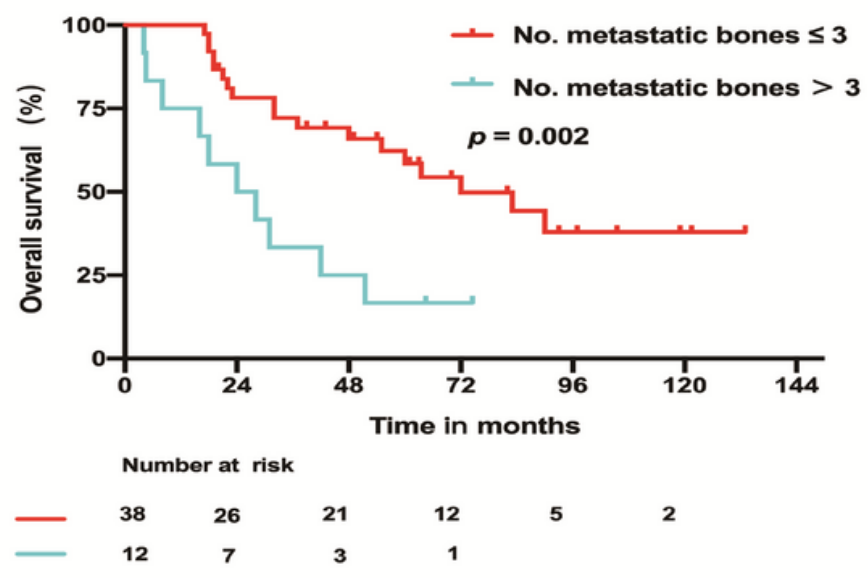

B

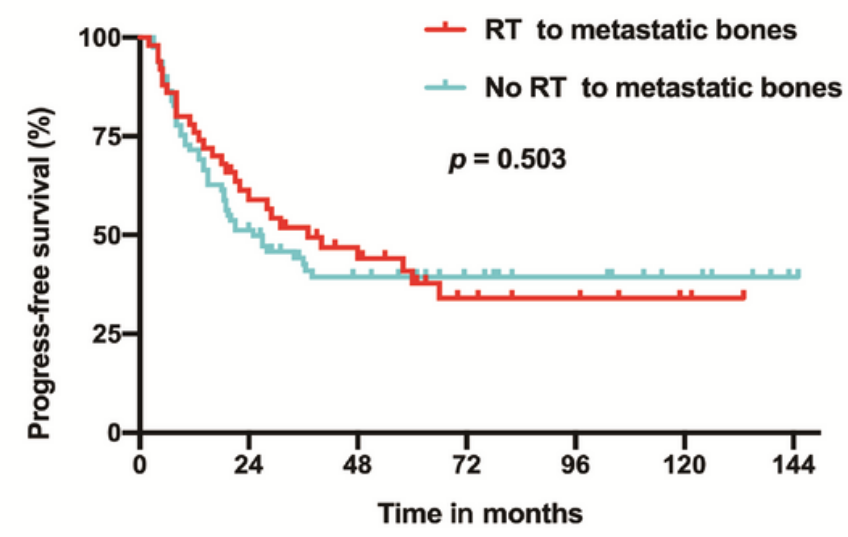

D

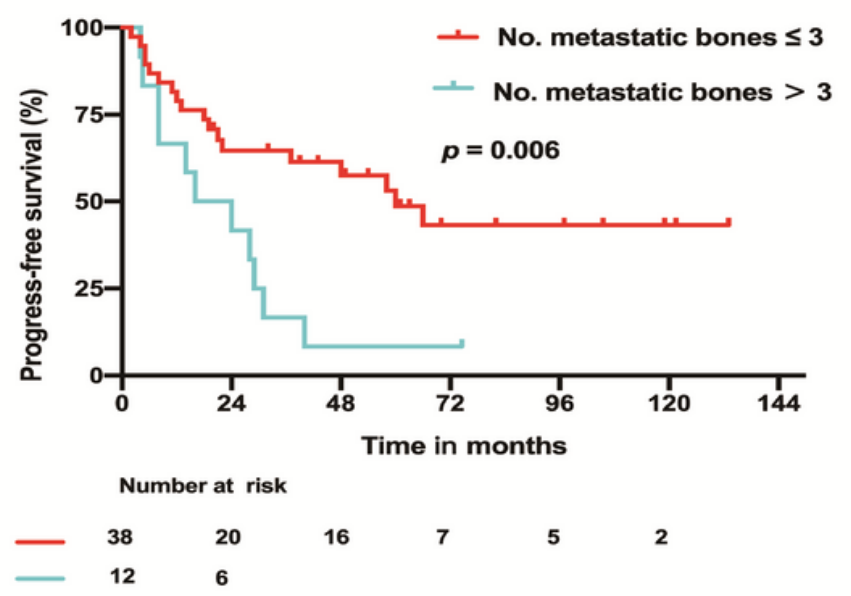

Figure 4

Kaplan-Meier curves for OS (a) and PFS (b) of 131 de novo mNPC patients based on whether patients received RT to metastatic bones or not; Kaplan-Meier curves according to No. of metastatic bones for OS (c) and PFS (d) of 50 de novo mNPC patients receiving PCT plus LLRT and RT to metastatic bones.

\section{Supplementary Files}

This is a list of supplementary files associated with this preprint. Click to download.

- TableS1.docx

- FigureS1.tif 